\title{
Dimerized phase and transitions in a spatially anisotropic square lattice antiferromagnet
}

\author{
Oleg A. Starykh ${ }^{1}$ and Leon Balents ${ }^{2}$ \\ ${ }^{1}$ Department of Physics and Astronomy, Hofstra University, Hempstead, NY 11549 \\ ${ }^{2}$ Department of Physics, University of California, Santa Barbara, CA 93106-4030
}

\begin{abstract}
We investigate the spatially anisotropic square lattice quantum antiferromagnet. The model describes isotropic spin-1/2 Heisenberg chains (exchange constant $J$ ) coupled antiferromagnetically in the transverse $\left(J_{\perp}\right)$ and diagonal $\left(J_{\times}\right)$, with respect to the chain, directions. Classically, the model admits two ordered ground states - with antiferromagnetic and ferromagnetic inter-chain spin correlations - separated by a first order phase transition at $J_{\perp}=2 J_{\times}$. We show that in the quantum model this transition splits into two, revealing an intermediate quantum-disordered columnar dimer phase, both in two dimensions and in a simpler two-leg ladder version. We describe quantum-critical points separating this spontaneously dimerized phase from classical ones.
\end{abstract}

PACS numbers:

An interplay between geometric frustration and quantum fluctuations is at the heart of intensive current investigations into the nature of possible $S U(2)$-invariant Mott insulators and quantum phase transitions. The absence of any "natural" small parameter, however, makes a traditional perturbative approach difficult. Aside from direct numerical attacks involving exact diagonalization and quantum Monte-Carlo techniques, this feature forces one to extend the parameter space of frustrated magnetic models and explore various "corners" of the resulting phase diagram in the hope of gaining new insights into the physically relevant region of system's parameters. In this paper, we explore a simple spatially anisotropic frustrated spin model which allows the application of powerful analytical methods borrowed from one dimension.

The model we consider - the spatially anisotropic square lattice antiferromagnet - is a quasi-onedimensional generalization of the well-studied square lattice antiferromagnet with frustrating antiferromagnetic exchange along diagonals, also known as the $J_{1}-J_{2}$ model. It describes a collection of antiferromagnetic spin- $S$ chains with exchange constant $J$ running along horizontal (chain) direction. The chains are interacting with their nearest neighbors via weak antiferromagnetic spin exchange in the transverse $\left(J_{\perp}\right)$ and diagonal $\left(J_{\times}\right)$, with respect to the chain, directions. Recently this model was investigated by Nersesyan and Tsvelik (NT) [1] who predicted a novel RVB-like phase with deconfined massive spinons at the special ratio of microscopic exchange constants: $J_{\perp}=2 J_{\times} \ll J$. We shall comment on this work and subsequent numerical investigations [11, 12] at the end of our paper. Let integer index $n$ numerate sites along the chain while index $m$ numerates chains. Then the Hamiltonian is $H=\sum_{m} H_{m}^{(0)}+V$, where $H_{m}^{(0)}$ is the standard Heisenberg Hamiltonian of the $m$-th chain and the interchain interaction reads

$$
V=\sum_{n, m}\left\{J_{\perp} S_{n, m}^{a} S_{n, m+1}^{a}+J_{\times} \sum_{q= \pm 1} S_{n, m}^{a} S_{n+q, m+1}^{a}\right\} \cdot(1)
$$

Here $a=x, y, z$ is the vector index over which implicit summation is implied. The classical $(S=\infty)$ phase diagram is simple: for $J_{\perp}>2 J_{\times}$ordering of the spins in the transverse to chain direction is antiferromagnetic (AFM phase), whereas for $J_{\perp}<2 J_{\times}$it is ferromagnetic (FM phase); spins order antiferromagnetically along chains. In the isotropic $J_{1}-J_{2}$ model these orderings correspond to the Néel and four-sublattice phases, respectively. The line $J_{\perp}=2 J_{\times}$describes a first order transition between these two phases. Along this line the interchain interaction can be written in a more suggestive form

$$
V_{\times}=\sum_{n, m} J_{\times}\left(S_{n, m}^{a}+S_{n+1, m}^{a}\right)\left(S_{n, m+1}^{a}+S_{n+1, m+1}^{a}\right) .
$$

Our goal is to understand the phase diagram of the quantum $S=1 / 2$ model in the limit of weak interchain couplings $J_{\perp}, J_{\times} \ll J$. Taking the continuum limit along the chain direction ( $x=n a, a$ is the lattice spacing), the spin operator is decomposed as

$$
S_{n, m}^{a} \rightarrow a\left(J_{m, R}^{a}(x)+J_{m, L}^{a}(x)+(-1)^{n} N_{m}^{a}(x)\right)
$$

in terms of chiral components $J_{m, R / L}^{a}$ of the uniform spin magnetization of the $m$-th chain, and the staggered spin magnetization $N_{m}^{a}$ (scaling dimension 1/2). The uniform spin magnetization $J_{m}^{a}=J_{m, R}^{a}+J_{m, L}^{a}$ is the conserved spin current (scaling dimension 1) and a generator of $O(3)$ spin rotations. The two fields in (3) are connected via the following operator product expansion (OPE)

$$
J_{R / L}^{a}(x, \tau) N^{b}\left(x^{\prime}, \tau^{\prime}\right)=\frac{\mp i \delta^{a b} \epsilon\left(x^{\prime}, \tau^{\prime}\right)+i \epsilon^{a b c} N^{c}\left(x^{\prime}, \tau^{\prime}\right)}{4 \pi\left[v\left(\tau-\tau^{\prime}\right) \mp i\left(x-x^{\prime}\right)\right]}
$$

Here the upper/lower signs on the right hand side apply for the right/left $(\mathrm{R} / \mathrm{L})$ moving currents, respectively, and $v=\pi J a / 2$ is the spin velocity. Of major importance is the appearance of the staggered dimerization operator $\epsilon$ (scaling dimension $1 / 2$ ). It is the continuum limit of the lattice operator involving the scalar product of two neighboring spins, $\epsilon_{m}(x) \sim(-1)^{n} S_{n, m}^{a} S_{n+1, m}^{a}$.

The spin algebra encoded in (4) and well-known OPE of the chiral components of the spin current 2] follow 
from the fact that the Hamiltonian density of the isolated Heisenberg chain is described by the $S U_{1}(2)$ WZW model perturbed by a marginally irrelevant backscattering term $\left(g_{\mathrm{bs}}=O(J)\right)$

$$
\mathcal{H}_{m}^{(0)}=\frac{2 \pi v}{3}\left(J_{m, R}^{a} J_{m, R}^{a}+J_{m, L}^{a} J_{m, L}^{a}\right)-g_{\mathrm{bs}} J_{m, R}^{a} J_{m, L}^{a} .
$$

The c-functions appearing in the OPE above are just the Green's functions of right- and left-moving fermions which are governed by the Hamiltonian (5) with $g_{\mathrm{bs}}=0$.

Having exposed the structure of the unperturbed theory, we now turn to the inter-chain interaction (1). Its continuum low-energy Hamiltonian density contains all terms allowed by the symmetries of the lattice model (these include reflection with respect to transverse direction and translation by one lattice constant)

$$
\begin{aligned}
\mathcal{V}= & \sum_{m} g_{1} N_{m}^{a} N_{m+1}^{a}+g_{2} J_{m}^{a} J_{m+1}^{a} \\
& +g_{3} a^{2} \partial_{x} N_{m}^{a} \partial_{x} N_{m+1}^{a}+g_{4} \epsilon_{m} \epsilon_{m+1}
\end{aligned}
$$

Here $\partial_{x}$ denotes derivative with respect to $x$ and the bare coupling constants follow from substituting (3) in (1)

$$
g_{1}=\left(J_{\perp}-2 J_{\times}\right) a, g_{2}=\left(J_{\perp}+2 J_{\times}\right) a, g_{3}=\frac{J_{\perp} a}{2}, g_{4}=0 .
$$

The scaling dimensions of the first, second, and third interaction terms in (6) are 1, 2, and 3, respectively. The last term (scaling dimension 1) is included here because it respects the symmetries of the lattice model (1). Although its bare coupling constant is zero, quantum fluctuations can (and will) generate some finite $g_{4}$.

At this level the phase diagrams of the quantum model, (6) with $g_{4}=0$, and classical one, (1) with $S=\infty$, are identical. As long as $J_{\perp} \neq 2 J_{\times}$, (6) is dominated by the strongly relevant interaction of staggered magnetizations which drives the system into one of the classically ordered phases: AFM for $g_{1}>0$ and FM for $g_{1}<0$.

However, the transitional region $J_{\perp} \approx 2 J_{\times}$requires a closer look because there the amplitudes of the both relevant terms are zero. To begin, let us fine-tune to the point $g_{1}=0$. On the lattice this corresponds to (2), whose continuum limit is given by (6) with $g_{1}=g_{4}=0$,

$$
\mathcal{V}_{\times}=\sum_{m} g_{2} J_{m}^{a} J_{m+1}^{a}+g_{3} a^{2} \partial_{x} N_{m}^{a} \partial_{x} N_{m+1}^{a}
$$

The situation is now apparently controlled by the marginally relevant $\left(g_{2}>0\right)$ current-current interaction which seems to suggest that the strongly irrelevant $g_{3^{-}}$ term does not play any role. Recall that the (KacMoody) algebra of spin currents $J_{R / L}^{a}$ is closed in the sense that OPE of two like currents produces another current of the same chirality 2]. Thus $\mathcal{V}_{\times}$with $g_{3}=0$ will "reproduce" itself in every order of the expansion in powers of the coupling constant $g_{2}$. This peculiar behavior is destroyed by $g_{3} \neq 0$ (or any other equally or even more irrelevant term). To see so, we expand the partition function to second order in $\mathcal{V}_{\times}$. As follows from the discussion above, one should concentrate on the cross-term $\left(\propto g_{2} g_{3}\right)$

$\int_{x, \tau} \int_{x^{\prime}, \tau^{\prime}} J_{m}^{a}\left(x^{\prime}, \tau^{\prime}\right) \partial_{x} N_{m}^{b}(x, \tau) J_{m+1}^{a}\left(x^{\prime}, \tau^{\prime}\right) \partial_{x} N_{m+1}^{b}(x, \tau)$.

Applying the OPE (4) to the same-chain operators at nearby points, differentiating the c-function pre-factors with respect to $x$ and performing elementary integration over the relative coordinates, one obtains a relevant correction to the Hamiltonian of the same form as (6). The coefficients in (77) are thereby replaced by

$$
g_{1}=\left(J_{\perp}-2 J_{\times}+\frac{2 J_{\times}^{2}}{\pi^{2} J}\right) a, g_{4}=-\frac{3 J_{\times}^{2} a}{\pi^{2} J},
$$

while two other couplings $g_{2,3}$ remain unchanged. Exactly the same result (9) can be obtained by performing the standard renormalization group (RG) analysis of (5) and (6). There one obtains five coupled non-linear differential equations for $g_{1-4}$ and $g_{\mathrm{bs}}$. In the transitional region, corresponding to $g_{1,4}=O\left(J_{\times}^{2} / J\right) \ll J_{\times}$, RG equations decouple and admit a simple analytical solution, which reproduces (9), for $\ell$ of the order 1 (here $\ell$ parametrizes the short-distance cutoff: $\left.a_{\ell}=a_{0} e^{\ell}\right)$. Our derivation of the renormalized coupling constants is very similar in spirit to the recent calculation of second-order corrections to coupling constants of two relevant and competing interactions in the extended Hubbard model 3].

What are the phases of $\mathcal{V}$ with relevant couplings given by (9)? We begin our analysis by studying two coupled chains, that is, frustrated spin ladder $(m=1,2$ in (6) $)$. Hamiltonian density of the ladder $\mathcal{H}_{\text {ladder }}=$ $\mathcal{H}_{1}^{(0)}+\mathcal{H}_{2}^{(0)}+\mathcal{V}$ can be recast as a theory of four massive real (Majorana) fermions, see Ch. 21 of [2],

$$
\begin{aligned}
\mathcal{H}_{\text {ladder }}= & \sum_{a=1}^{4}\left(-\frac{i v}{2} \xi_{R}^{a} \partial_{x} \xi_{R}^{a}+\frac{i v}{2} \xi_{L}^{a} \partial_{x} \xi_{L}^{a}\right) \\
& -i m_{t} \sum_{a=1}^{3} \xi_{R}^{a} \xi_{L}^{a}-i m_{s} \xi_{R}^{4} \xi_{L}^{4}+\delta \mathcal{H}_{\text {marg }}
\end{aligned}
$$

where $\delta \mathcal{H}_{\text {marg }}$, originating from $g_{\mathrm{bs}}$ in (5) and $g_{2}$ in (6), describes residual marginal interactions between Majorana fermions

$$
\delta \mathcal{H}_{\text {marg }}=\frac{g_{-}}{4}\left(\sum_{a=1}^{3} \xi_{R}^{a} \xi_{L}^{a}\right)^{2}-\frac{g_{+}}{2} \sum_{a=1}^{3} \xi_{R}^{a} \xi_{L}^{a} \xi_{R}^{4} \xi_{L}^{4},
$$

with $g_{ \pm}=g_{2} \pm g_{\mathrm{bs}}$. Ignoring $\delta \mathcal{H}_{\text {marg }}$, the first three Majorana fermions form a triplet with the mass

$$
m_{t}=\frac{\lambda^{2}\left(g_{1}-g_{4}\right)}{2 \pi a}=\frac{\lambda^{2}}{2 \pi}\left(J_{\perp}-2 J_{\times}+\frac{5 J_{\times}^{2}}{\pi^{2} J}\right),
$$

whereas the fourth fermion $\xi_{R / L}^{4}$ is a singlet with

$$
m_{s}=-\frac{\lambda^{2}\left(3 g_{1}+g_{4}\right)}{2 \pi a}=-\frac{3 \lambda^{2}}{2 \pi}\left(J_{\perp}-2 J_{\times}+\frac{J_{\times}^{2}}{\pi^{2} J}\right) .
$$


Both masses receive finite logarithmic corrections from $\delta \mathcal{H}_{\text {marg }}$ which we omit in the following. Here $\lambda \sim 1$ is the expectation value of the charge operator which multiplies abelian bosonization expressions for the staggered magnetization and dimerization operators [4]

$$
\begin{aligned}
\mathbf{N}_{m} & =\frac{\lambda}{\pi a}\left(\cos \sqrt{2 \pi} \theta_{m}, \sin \sqrt{2 \pi} \theta_{m},-\sin \sqrt{2 \pi} \varphi_{m}\right) \\
\epsilon_{m} & =\frac{\lambda}{\pi a} \cos \sqrt{2 \pi} \varphi_{m}
\end{aligned}
$$

The Hamiltonian (10) predicts two transitions [5] which happen when one of the renormalized masses becomes zero.

(i) $m_{t}=0$ : at this point triplet excitations become gapless. Integrating out massive fermion $\xi^{4}$, we find that $\mathcal{H}_{\text {ladder }}$ is the Hamiltonian of the $S U_{2}(2)$ WZW model with central charge $c=3 / 2$. The nature of the transition is determined by the sign of remaining marginal coupling $g_{2}-g_{\text {bs }}$. Its positive initial value implies marginally relevant flow to the strong coupling, and hence, first order phase transition. For the negative initial value one obtains marginally irrelevant flow of the coupling constant to zero, and hence, second order transition.

(ii) $m_{s}=0$ : singlet excitations become massless. This is a continuous $Z_{2}$ (Ising) transition.

Properties of the various phases are most conveniently understood from the bosonized form of the relevant part of interchain interaction (6), $\mathcal{V}_{\text {rel }}$, which corresponds to mass terms in (10). In terms of symmetric and antisymmetric combinations of bosonic fields on two chains, $\varphi_{ \pm}=\left(\varphi_{1} \pm \varphi_{2}\right) / \sqrt{2}$ (and similarly for $\theta_{ \pm}$) it reads

$$
\begin{aligned}
\mathcal{V}_{\text {rel }}= & \frac{\lambda^{2}}{2(\pi a)^{2}}\left(2 g_{1} \cos \sqrt{4 \pi} \theta_{-}+\left(g_{1}+g_{4}\right) \cos \sqrt{4 \pi} \varphi_{-}\right. \\
& \left.-\left(g_{1}-g_{4}\right) \cos \sqrt{4 \pi} \varphi_{+}\right)
\end{aligned}
$$

Depending on the ratio $g_{1} / g_{4}$ four phases are possible: (a) $g_{1} \rightarrow-\infty$ is the Haldane (effective spin- 1 chain) phase; $m_{t}<0, m_{s}>0$ and $\varphi_{+}=\sqrt{\pi} / 2, \theta_{-}=0$. Inter-chain spin correlations are ferromagnetic. This is the ladder analog of the classical FM phase.

(b) $g_{1} \rightarrow+\infty$ is the rung-singlet phase; $m_{t}>0, m_{s}<0$ and $\varphi_{+}=0, \theta_{-}=\sqrt{\pi} / 2$. Here inter-chain spin correlations are antiferromagnetic, which corresponds to the classical AFM phase.

(c) $g_{4} \rightarrow-\infty$ is the columnar dimer phase (DC); $m_{s, t}>0$ and $\varphi_{+}=0, \varphi_{-}=0$.

(d) $g_{4} \rightarrow+\infty$ is the staggered dimer phase (DS); $m_{s, t}<0$ and $\varphi_{+}=\sqrt{\pi} / 2, \varphi_{-}=\sqrt{\pi} / 2$.

The last two phases are spontaneously dimerized and do not have classical prototypes. Using (14) we find that both dimerized phases break translational symmetry (along the chain direction) but their ordering patterns are different. In the DC phase $\left\langle\epsilon_{1}\right\rangle=\left\langle\epsilon_{2}\right\rangle$, which describes columnar dimer long-range order. In the DS phase $\left\langle\epsilon_{1}\right\rangle=-\left\langle\epsilon_{2}\right\rangle$, which makes it the staggered dimer state. Observe that microscopic couplings (9) choose

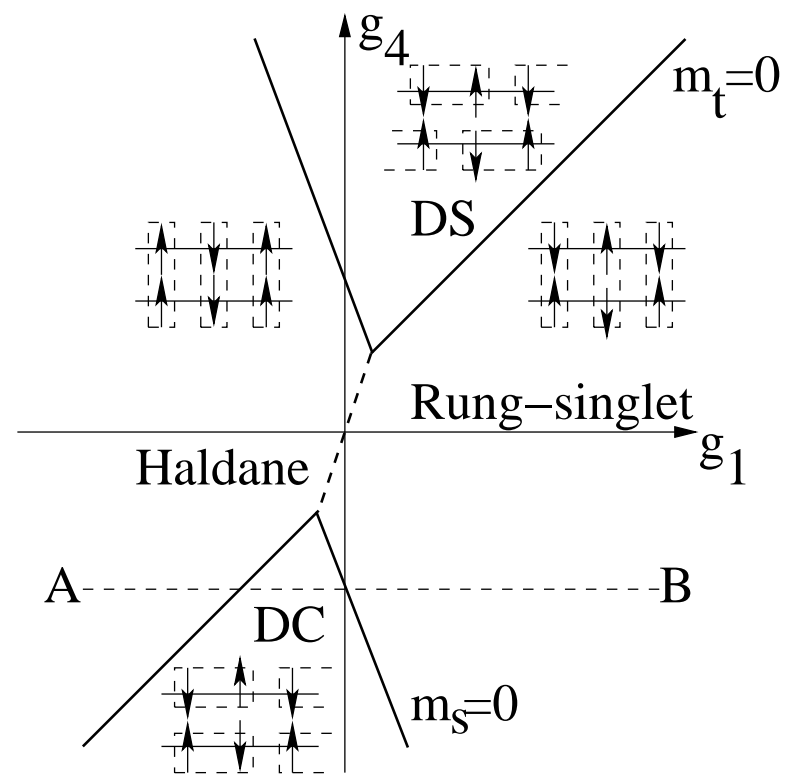

FIG. 1: Phase diagram of the frustrated ladder. Line AB marks the parameters corresponding to the lattice model.

columnar dimer as the intermediate phase between the Haldane and rung-singlet phases of the frustrated ladder.

This feature was missed by [6, 7] who described $J_{\perp}=$ $2 J_{\times}$point of the lattice ladder by (8) with $g_{3}=0$. Our analysis shows that multicritical point where chains are coupled only by the marginal current-current interaction requires additional fine-tuning of both $g_{1}, g_{4}$ (equally, of $\left.m_{s}, m_{t}\right)$ to zero. A more detailed understanding can be gained by considering the model $g_{2}>0$ and exponentially small relevant couplings $g_{1,4}$ (more specifically $\left.1 /\left|\ln g_{1,4}\right| \ll g_{2}\right)$ - though this is not realized by the lattice model. In this case the NT analysis [1] can be taken over, treating $g_{1,4}$ as small perturbations. A semiclassical analysis for $g_{1,4}=0$ shows that the NT model has a manifold of ground states in which minima of type (a) and (b) above - i.e. Haldane and rung-singlet states are degenerate. Perturbing with (15) above splits the energy densities of these two degenerate states, so that the $g_{4}=3 g_{1}$ becomes a first order transition line (dashed line through the origin in Fig. 11. Furthermore, the spinons of the NT phase correspond to domain walls between these two coexisting states. Remembering that the finite length spin chains in the Haldane phase are characterized by spin- $1 / 2$ end states, the spinons become physically transparent as mobile versions of these. Away from the first order line, such spinons are therefore confined (i.e. cost an energy linear in system size), due to the energy cost to create a domain of the disfavored phase. Thus although it does not occur in the lattice model studied here, the NT state and its spinons can exist as a first order line in a generalized ladder model.

Having understood the ladder, we tackle the full twodimensional problem. We treat the relevant inter-chain interactions $g_{1,4}$ (taking $g_{2,3}=0$ ) using a self-consistent 
mean-field approximation [8, [9]. Assuming symmetry breaking along $z$-direction,

$$
\mathcal{V}=-z\left(\left|g_{1}\right|\left\langle N_{m}^{z}\right\rangle N_{m}^{z}+\left|g_{4}\right|\left\langle\epsilon_{m}\right\rangle \epsilon_{m}\right)
$$

where we used $\left\langle N_{m+1}^{z}\right\rangle= \pm\left\langle N_{m}^{z}\right\rangle$ for $g_{1}$ negative (positive), and $z=2$ is the number of neighboring chains. Using (14) and bosonized form of $\mathcal{H}_{m}^{(0)}$ (with $g_{\mathrm{bs}}=0$ ), rescaling euclidian time $\tau=y / v$ and introducing dimensionless euclidian distance $r=(x, y) / a$, we arrive at the following single-chain sine-Gordon action

$$
S_{\mathrm{mf}}=\int d^{2} r\left(\frac{1}{2}\left(\nabla_{r} \varphi\right)^{2}-p \sin \sqrt{2 \pi} \varphi-q \cos \sqrt{2 \pi} \varphi\right)
$$

where dimensionless parameters

$$
p \equiv \frac{z \lambda^{2}\left|g_{1}\right|}{\pi^{2} v}\langle\sin \sqrt{2 \pi} \varphi\rangle, \quad q \equiv \frac{z \lambda^{2}\left|g_{4}\right|}{\pi^{2} v}\langle\cos \sqrt{2 \pi} \varphi\rangle
$$

are effective staggered magnetization and dimerization fields, respectively. These averages are found by differentiating the free energy density $F(p, q)=-\ln Z / V, Z=$ $\operatorname{Tr}\left(e^{-S_{\mathrm{mf}}}\right)$ with respect to $p$ and $q$, respectively. Clearly from (17), the mean-field free energy can depend on $p, q$ only through $\kappa=\sqrt{p^{2}+q^{2}}$. One can therefore take $F(p, q)=F(0, \kappa)$ and take advantage of exact results for the standard sine-Gordon action [10]. In particular $d F / d \kappa=-c_{1} \kappa^{1 / 3}$, where the numerical constant $c_{1}$ follows from equations (10-14) of ref. 10]

$$
c_{1}=\frac{\tan (\pi / 6)}{3}\left(\frac{2 \Gamma(1 / 6)}{\sqrt{\pi} \Gamma(2 / 3)}\right)^{2}\left(\frac{\pi \Gamma(3 / 4)}{2 \Gamma(1 / 4)}\right)^{4 / 3} .
$$

Thus our self-consistent equations

$$
\langle\sin \sqrt{2 \pi} \varphi\rangle=\frac{c_{1} p}{\kappa^{1 / 3}}, \quad\langle\cos \sqrt{2 \pi} \varphi\rangle=\frac{c_{1} q}{\kappa^{1 / 3}}
$$

become simple algebraic ones. They predict two transitions, at $g_{1}= \pm\left|g_{4}\right|$, where both order parameters $\left(N_{m}^{z}, \epsilon_{m} \sim p, q\right)$ are non-zero. This makes them firstorder transitions - the first order nature being likely an artifact of the mean-field approximation. These two transitions separate three phases, indicated in Fig. 2 For $\left|g_{1}\right|>\left|g_{4}\right|, p \neq 0, q=0$, so the system is AFM or FM, depending on the sign of $g_{1}$. For $\left|g_{1}\right|<\left|g_{4}\right|$, one finds the columnar dimer phase (rather than staggered since $\left.g_{4}<0\right)$. Note that obtained phase diagram (Fig. (2) is in qualitative agreement with that of the ladder, which provides a posteriori support of our mean-field treatment. Moreover, it agrees very well with the phase diagram of the lattice model (11) obtained in the exact diagonalization study 12. We should add that DC phase was not observed in DMRG study [11], presumably due to strong finite-size effects at small $J_{\perp} / J$. Now, since exactly this type of dimer ordering is known to take place in the spatially isotropic $J_{1}-J_{2}$ model [13], it is natural to conclude that the DC phase found here extends all way up to $J_{\perp}=J$.

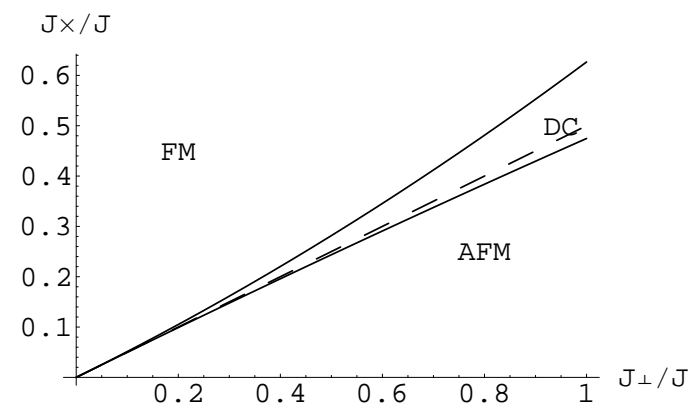

FIG. 2: Phase diagram of the two-dimensional model. Solid (dashed) lines denote phase boundaries of the quantum (classical) model.

We thank the Aspen Center for Physics, where this work has began, and the Kavli Institute for Theoretical Physics, where it continued, for hospitality. We are grateful to A. Furusaki and A. Vishwanath for many stimulating discussions. O.A.S. thanks A. Abanov, F. Essler, P. Lecheminant and A. Tsvelik for useful discussions at the Theory Institute of the Brookhaven National Laboratory, and, especially, A. Nersesyan for illuminating discussion of the OPE (47). This research was supported by NSF Grant No. PHY99-07949 (O.A.S.), DMR-9985255 (L.B.), the Research Corporation Grant No. CC5491 (O.A.S.) and the Packard Foundation (L.B.).
[1] A. A. Nersesyan and A. M. Tsvelik, Phys. Rev. B 67 024422 (2003).

[2] A. O. Gogolin, A. A. Nersesyan, and A. M. Tsvelik, Bosonization and strongly correlated systems, Cambridge University Press (1998).

[3] M. Tsuchiizu and A. Furusaki, Phys. Rev. Lett. 88 056402 (2002), and Phys. Rev. B 69, 035103 (2004).

[4] D. G. Shelton, A. A. Nersesyan and A. M. Tsvelik, Phys. Rev. B 53, 8521 (1996).

[5] A. A. Nersesyan and A. M. Tsvelik, Phys. Rev. Lett. 78, 3939 (1997).

[6] D. Allen, F. H. L. Essler, and A. A. Nersesyan, Phys.
Rev. B 61, 8871 (2000).

[7] E. H. Kim, G. Fáth, J. Sólyom, and D. J. Scalapino, Phys. Rev. B 62, 14965 (2000).

[8] H. Schulz, Phys. Rev. Lett. 77, 2790 (1996).

[9] F. H. L. Essler, A. M. Tsvelik, and G. Delfino, Phys. Rev. B 56, 11001 (1997).

[10] S. Lukyanov and A. Zamolodchikov, Nucl. Phys. B 493, 571 (1997); hep-th/9611238

[11] S. Moukouri, cond-mat/0305608

[12] P. Sindzingre, cond-mat/0307118

[13] N. Read and S. Sachdev, Phys. Rev. B 424568 (1990). 